\title{
PLANNING OF BED CAPACITIES IN SPECIALIZED AND INTEGRATED CARE UNITS: INCORPORATING BED BLOCKERS IN A SIMULATION OF SURGICAL THROUGHPUT
}

\author{
Navonil Mustafee \\ Swansea University \\ School of Business and Economics \\ Swansea SA2 8PP, Wales, UK \\ Terry Lyons \\ University of Oxford \\ Mathematical Institute, 24-29 St Giles' \\ Oxford OX1 3LB, UK
}

Paul Rees

Abertawe Bro Morgannwg University Health Board

Cardiac Centre, Morriston Hospital

Swansea SA6 6NL, Wales, UK

\author{
Lee Davies \\ NHS Wales \\ Delivery and Support Unit, Bocam Park, Pencoed \\ Bridgend CF35 5LJ, Wales, UK
}

Mark Ramsey

Abertawe Bro Morgannwg University Health Board

Cardiac Centre, Morriston Hospital

Swansea SA6 6NL, Wales, UK

Michael D. Williams

Swansea University

School of Business and Economics

Swansea SA2 8PP, Wales, UK

\begin{abstract}
Simulation has been applied for the management of bed capacities in hospitals. However, the majority of these studies have ignored the application of this technique in specalized and integrated care units, wherein different wards, e.g., general ward, Intensive Therapy Unit (ITU), High Dependency Unit (HDU), are organized to provide patients differing levels of care as they progress through the treatment pathway. In this set-up, bed blocking occurs when patients that are clinically ready to be discharged from wards that provide higher levels of care (e.g., ITU) cannot be transferred to wards offering reduced care (e.g., HDU, general ward) because of the non-availability of beds in the latter wards. This has implications on throughput of clinical activity, as well as patients' cost of treatment. In this paper we investigate this problem of bed blocking through a case study being conducted at the Cardiac Intensive Care Unit at Morriston Hospital, Wales (UK).
\end{abstract}

\section{INTRODUCTION}

Discrete event simulation (DES) is an operations research (OR) technique that is widely used to model queuing systems. The use of DES permits the stakeholders to create computer representation of systems under scrutiny and to model their underlying behavior over a simulated time frame. The DES methodology lays particular emphasis on detailed-level modeling wherein it attempts to imitate the observed behavior of a system through time by (a) conceptualizing it as networks of queues and servers, and (b) using stochastic distributions to generate events and quantities typical of the real system being modeled (Chahal and Eldabi 2008). Monte Carlo Simulation (MCS) is yet another simulation technique which uses a sequence of random numbers to generate values from a known probability distribution associated with a 
source of uncertainty (Rubinstein 1981). Although both MCS and DES are stochastic in nature there is a key difference between them - MCS is an example of a static system model (i.e., time is not a significant variable), whereas DES is a dynamic model in which the passage of time is a significant factor since the time evolution of the system state variables is important (Leemis and Park 1994). The work presented in this paper is related to both MCS and DES.

Computer simulation has several advantages, e.g., it is cheaper than building (and discarding) real systems; they assist in the identification of bottlenecks and allow for testing of alternate strategies; they allow for faster than real-time experimentation; and they involve lower costs compared to experimentation with real systems (Brooks, Robinson, and Lewis 2001; Pidd 2004). It therefore comes as no surprise that computer simulation is widely used in the industry as a decision support tool; its application in the context of healthcare operations management is also on the rise. It is arguable that the ongoing global financial and economic crisis (2007-present) that has seen governments striving to provide better healthcare services at a lesser cost (e.g., through efficiency savings - Nicolson's Challenge) and in an environment of increasing citizens' expectations, will further stimulate interest for simulation-based studies since it necessitates the careful use of the allocated healthcare budgets through better and more informed decision making (Mustafee et al. 2011).

The paper presents an ongoing study that uses MCS and DES for bed capacity planning at the Cardiac Intensive Care Unit (CITU), which is a part of Morriston Hospital (MH) Cardiac Centre. The patients in need of cardiac surgery are admitted to CITU. An investigation of the system in question has identified bed blocking (lack of availability of beds further along the clinical pathway) to be a significant constraint that is preventing an increased throughput of patients through CITU; this is consequently affecting the number of cardiac surgeries being conducted by the Cardiac Centre. The question that we ask ourselves at this juncture is, will an increase in the number of beds reduce or indeed eliminate bed blocking? Or are further strategies required to reduce the impact of this bottleneck? To answer these questions we identify two objectives of this study - (a) stakeholder objective and (b) research objective. The stakeholder objective is based on the premise that there is a shortage of beds, and it therefore concerns with determining the optimal bed configuration that would enable the Centre to increase the number of cardiac surgeries; the realization of this objective would contribute to local impact. The research objective takes a wider perspective, and through the use of the CITU case study, it aims to investigate bed management strategies that attempt to reduce bed blocking in specialized and integrated care units that operate in large parts as a closed-system (as is the case with CITU). The underlying premise here is that the number of beds cannot be increased in the short run. Also, an increase in bed numbers in the long term may need to be complemented with bed management strategies if we were to derive maximum efficient from the system. Although two objectives have been defined in this paper, the results presented relate to the stakeholder objective. The work related to the fulfillment of the research objective is ongoing work.

The remainder of the paper is organized as follows. Section 2 provides the context of this work and further elucidates its motivation. Section 3 presents a review of literature focusing on studies that have used MCS and DES for modeling bed capacities. The CITU case study is presented in Section 4. The study has led to the development of a MCS and a DES model. Section 5 describes the MCS and presents some results. Section 6 argues the need for DES to model the detailed interactions in the underlying system as this would enable experimenting with bed management strategies to reduce bed blocking. This section also presents the DES model which is currently under development. Section 7 is the concluding section of this paper. It underlines the future work and draws the paper to a close.

\section{CONTEXT AND MOTIVATION}

\subsection{Stakeholder objective}

The Heath Board is contracted by the NHS to perform 820 cardiac operations every year comprising a mix of in-house urgent and emergency operations (approximately 50\% of activity) and planned elective operations from a waiting list. The Health Board is required to deliver timely surgery for both groups of 


\section{Mustafee, Davies, Lyons, Ramsey, Rees, and Williams}

patients. Those on the waiting list are expected to have a maximum referral (from the GP) to treatment time (cardiac surgery) of no more than 26 weeks. Statistics from December 2011 show that about 100 patients on the waiting list have waited more than 36 weeks. Data from 1st April 2009 to 31st March 2010 show that a total of 707 cardiac operations were performed during this period (ABM UHB INFO 2010). Non-availability of beds has been identified to be a significant constraint (accounting for about $80 \%$ of cancelled operations) that is preventing the Health Board from increasing surgical activity. Therefore, the work intends to provide the cost-benefit analysis of increasing throughput through changes to bed configuration (considering both increased bed numbers and alternative bed arrangements). The MCS and the DES models (described in Sections 5 and 6 respectively) have been developed for conducting this analysis.

\subsection{Research objective}

In specialized and integrated care units like CITU, hospital beds may be organized into wards that provide patients differing levels of care (Level $0 / 1,2,3$ ) as they progress through the treatment pathway. Level $0 / 1$ care is for patients whose needs can be met through normal ward or those at risk of their condition deteriorating; Level 2 care is for patients that require more detailed observation and intervention, including access for post-operative care; Level 3 care is provided to patients that require either advance or basic respiratory support, together with support for at least two organ systems (ICS 2011). Consistent with the aforementioned levels of care, the beds are generally organized into general wards for Level 0/1 care, HDU for Level 2 care and ITU for Level 3 care. In this set-up, bed blocking occurs when patients that are clinically ready to be discharged from wards that provide higher levels of care (e.g., ITU) cannot be transferred to wards offering reduced care (e.g., HDU, general ward) because of the non-availability of beds in the latter wards.

Bed blocking contributes to the increase in the patients' cost of treatment. The cost of care in ITU is higher compared to the cost of care in HDU and general ward, and therefore, if bed blocking prevents transfer of patients between wards then this translates to the comparative higher treatment costs for the health service for each night of patients' stay in wards offering higher-level of care. This additional cost could have been avoided for patients that were clinically ready to be transferred to wards offering reduced levels of care. The work therefore intends to propose strategies to reduce the economic burden of treatment. The DES model (Section 6) will enable the stakeholders to experiment with such strategies.

\section{RELATED WORK}

There are several studies that have reviewed the application of simulation in the context of healthcare (Katsaliaki and Mustafee 2011; Brailsford et al. 2009; Fone et al. 2003; Jun, Jacobson, and Swisher 1999). For example, through a methodological review of literature published in impact-factor journals, the study by Katsaliaki and Mustafee (2011) identified 177 studies that applied MCS and DES for planning of healthcare services (e.g., planning of hospitals and A\&E departments, scheduling health staff, modeling bed and equipment capacity, organ transplantation, locations of healthcare services and facilities design), health economic modeling (e.g., cost of providing healthcare, alternative healthcare interventions, screening strategies), contagious disease interventions (e.g., controlling the spread of diseases/epidemics, planning of emergency clinics), health risk assessment (e.g., drug development-dose response), etc. The bed capacity modeling work presented in this paper is related to one such application category, namely, planning of health services.

Bagust, Place, and Posnett (1999) have used DES to examine the dynamics of bed use arising from the flow of emergency admissions in an hypothetical setting. The effect of bed numbers on the flow of patients in a geriatric department has been investigated by El-Darzi et al. (1998) through mixed exponential modeling (using Bed Occupancy Modeling and Planning System) and queuing networks (using Micro Saint DES package). These simulation studies have mainly reported on the effects of bed blocking. However, the purpose of the present study is to plan for the future by experimenting with optimal bed configu- 
ration and strategies for bed management. The work by Heslop et al. (2004) refer to the development of a bed management strategy and modeling of a new HDU ward (in contrast to maintaining patients requiring HDU care in ITU) at Peninsula Health, a health care provider serving Victoria's Mornington Peninsula, Australia. However, the aforementioned paper is on patient data management and not specifically on simulation, and thus no further detail of this work is provided (also, there are no references related to the modeling work). The impetus for the work by Utley et al. (2003, pp. 1) complements the stated motivation for our paper, and we quote from Utley et al. (2003) "Concern has been expressed in the United Kingdom regarding the proportion of beds intended for acute care that are occupied by patients who do not require acute care"; however, the focus of the paper is on the use of mathematical modeling to facilitate informed decision making pertaining to the building of intermediate care facilities for non-acute care. Thus, this study is not specifically concerned with bed blocking which might arise when patients have to be transferred from acute care to non-acute care.

There are several other survey-based studies that have examined the effect of bed blocking in specific contexts, for example, Rubin and Davies (1975) conducted a survey in three general hospitals to investigate the problem of elderly patients blocking beds that are meant for acute care; the study by Namdaran, Burnet, and Munroe (1992) focused on a survey of in-patients in all acute hospitals within the city of Edinburgh (UK); the survey study by Coid and Crome (1986) aimed to ascertain the extent of bed blocking in Bromley (in Greater London) and to suggest ways to minimize this problem. These papers provide further empirical evidence of the wide spread existence of bed blocking in hospital in the UK.

\section{CASE STUDY}

\subsection{The Cardiac Centre at Morriston Hospital}

Abertawe Bro Morgannwg (ABM) University Health Board (UHB) is amongst the largest health boards in Wales. It has around 17,000 staff serving a population of approximately 600,000 and covering the areas of Bridgend, Neath Port Talbot and Swansea (ABMUHB 2012). Morriston Hospital (MH) is the only hospital under the ABM UHB that has facilities for cardiac surgery; it is one of only two hospitals in Wales that perform open-heart surgeries (the other being University of Wales Hospital, Cardiff). Following cardiac surgery, all patients are managed initially in the CITU located in the MH Cardiac Centre. The Centre commenced operations in 1997 and provides tertiary cardiac services (including cardiology and cardiothoracic surgery) to the resident population in its catchment area.

The performance of the cardiac centers in Wales is noteworthy. In a survey conducted by the European Association for Cardiothoracic Surgery (EACS), data pertaining to approximately one million cardiac surgical operations performed in 23 European countries were analysed and it was concluded that Wales outperformed England and most of Europe with regard to survival rates for patients undergoing coronary artery bypass graft surgery; Wales had a mortality rate of $1.1 \%$, compared to $1.8 \%$ in England and 2.4\% in Europe (RCS 2010).

\subsection{CARDIAC INTENSIVE CARE UNIT (CITU)}

Patients in need of cardiac surgery are admitted to CITU. It has two dedicated theatres for cardiac surgery and three wards, namely Cyril Evans Ward (CEW), HDU, and ITU. CEW has 25 beds and is for Level $0 / 1$ care; ITU has eight beds and is for immediate post-operation recovery (Level 3 care); HDU also has eight beds and is used for Level 2 care.

\subsubsection{Ward Staffing}

During the weekdays the ITU and HDU beds are manned by a total of 13 nurses. Nurse-to-Bed ratio is $1: 1$ for ITU and 1:2 for HDU - thus, a total of 12 nurses are usually required for the 16 ITU and HDU beds. In addition, one nurse is assigned for managing the nursing operations. From Saturday 1200 to Monday 


\section{Mustafee, Davies, Lyons, Ramsey, Rees, and Williams}

0900 the Cardiac Centre is funded for only nine nurses and this reduces the total number of ITU and/or HDU beds available for cardiac surgery patients. The total pool of eight nurses (note, the ninth nurse is required for management) can be allocated between the ITU and the HDU wards depending upon the clinical requirement of the patients staying in the respective wards. For example, it would be possible to provide care for six ITU patients and four HDU patients, or four ITU patients and eight HDU patients. The number of beds available in CEW remains unchanged during the weekend ( 25 beds).

\subsubsection{Operating Theatre}

There are two dedicated theatres and five consultant cardiac surgeons. The theatres usually operate Monday to Friday from 0830 to 1900. Cardiac surgeries can take anywhere between four to six hours (this excludes the time required to set-up the theatres) and therefore each theatre can usually perform a maximum of two cardiac surgeries each day. Considering 252 working days in a year, we can calculate the total cardiac surgery capacity at CITU to be around 1008 - this is the best case scenario. However, the actual number of surgeries that can be performed (even at $100 \%$ theatre capacity utilization) is lower than this number since working days are lost for the purposes of internal audit. Also, cardiac surgeries can be performed only if there are patients waiting for the surgery. A $100 \%$ capacity utilization assumes the existence of a queue of patients that could be operated on; however, this is not always the case. A $100 \%$ capacity utilization also assumes the availability of ITU beds for patients subsequent to the surgery. Again, this cannot be guaranteed due to bed blocking.

\subsection{Flow of Patients through the System}

The discussion presented in this section focuses on the flow of patients that are in the elective patients' waiting list. They do not take into consideration the prospect of private patients being operated during the weekends (this happens very infrequently). However, it should be noted here that private patients do have an impact on ITU, HDU and CEW bed availability.

Cardiac surgery patients from the elective patients' waiting list are admitted to the CEW usually one day prior to the surgery for pre-operative examination and tests. Subsequent to the surgery the patients are moved to the ITU for immediate post-operative care. The average length of stay in ITU is between 12-24 hours (MCC 2006). Patients are transferred to the HDU when the consultants are satisfied with the patients' post-operative recovery in ITU. From HDU the patients are transferred back to the CEW, where they are prepared for discharge. Although the movement of the patients through the three CITU wards is mostly linear $(\mathrm{CEW} \rightarrow \mathrm{ITU} \rightarrow \mathrm{HDU} \rightarrow \mathrm{CEW})$, there may also be considerable deviation caused by nonavailability of beds further down the process chain (e.g., transfer of patients between hospitals may become necessary due to non-availability of HDU beds; patients may be transferred to MH ITU ward since the ITU ward of CITU may be full to capacity). It is arguable that this patient transfer arrangement, both intra- and inter-hospital, is unsatisfactory since the cardiologists would like to monitor the patients they operate on and see them through to full recovery.

\subsubsection{Length of Stay}

The "Pre-Operative Information Booklet for Cardiac Patients" (MCC 2006) states that the patients are ready for discharge in about 5-6 days following the cardiac surgery. However, there is considerable variation between patients with regard to the total hospital stay in the hospital and also the length of stay (LOS) in the individual wards. For example, analysis of historic data relating to patients' LOS in ITU reveals that although the median value of patients' stay is 1785 minutes (around 30 hours), the average LoS is 4674 minutes (3.24 days). One reason for this is that a minority of patients may require Level 3 care for a few weeks, and this increases the average (for example, one cardiac surgery patient required 143.54 days in ITU). The data that was used for this analysis was ward stay data from March 2009-October 2010. The analysis was performed using Stat:Fit. 


\subsubsection{Shared Services}

Some CITU resources are shared with patients for thoracic surgery. Although these surgeries are not performed in the cardiac surgery operating theatres, the patients that have undergone this surgery are moved to HDU for Level 2 care, and onwards to CEW. Very few patients are however moved to the ITU (only 26 patients were transferred during the period 1st April 2009 to 31st October 2010; this includes patients that may have been transferred from HDU to ITU due to deterioration in the their medical condition). The thoracic surgeries take place on Tuesdays and Thursdays and a maximum of eight surgeries are performed every week. Considering the average length of HDU stay of 2-3 days, it can be argued that the thoracic surgeries taking place on Tuesday affect bed availability for cardiac surgery patients on Tuesday (from 1200), Wednesday, and Thursdays (until 1200). Similarly, thoracic surgeries taking place on a Thursday affect bed availability for cardiac surgery patients on Thursday (from 1200), Friday, and Saturday (until 1200). Since the number of patients undergoing thoracic surgery in the Cardiac Centre is on the rise, it is expected that competition for resources (primarily, HDU and ITU beds) among the two cardiac subspecialties will to be more intense in the near future.

\subsection{Bed blocking in CITU}

Beds are frequently unavailable in CITU due to the occupants being too unwell to be discharged over many days (so-called "long-stay" patients) or because of bed blocking. On any given day, bed blocking has a direct bearing on the number of cardiac operations that can be performed in CITU since the availability of an ITU bed is a necessary pre-condition for performing a cardiac operation. As the flow of patients through the three CITU wards is mostly linear (CEW $\rightarrow$ ITU $\rightarrow$ HDU $\rightarrow$ CEW), it therefore follows that bed blocking observed down the process chain (e.g., non-availability of beds in HDU) will affect transfer of patients from the preceding wards (e.g., patient from ITU cannot be transferred to HDU), which, in turn, will affect the number of cardiac operations being performed.

\section{MODELLING BED CAPACITY USING MONTE CARLO SIMULATION}

The MCS model is only concerned with the ITU and the HDU elements of the Cardiac Centre and therefore assumes the level of bed blocking remains constant. The Excel-based MCS was implemented during the development phase of the CITU DES model (Section 6), with the former being intentionally simplified as the latter was being developed to model the detailed interactions existing in the underlying system. With regard to the MCS, the length of stay data (March 2009 - October 2010) for the combined ITU and HDU is utilized to simulate bed stay requirements based on varying activity scenarios. Previous analysis of length of stay distributions over the past three financial years demonstrated no change over this period (over longer periods it is believed length of stay in ITU/HDU has increased and become more variable) so it was assumed the distribution of the length of stay dataset would adequately represent future bed requirements at least over the short to medium term (1-3 years).

The MCS models the impact of varying theatre schedules and varying bed capacity on the achieved activity levels (surgical procedures undertaken) and cancellation rates due to a lack of beds (in the realworld scenario cancellations may occasionally occur for other reasons, infection outbreaks or surgeon unavailability for example, but these are not factored into the model). Patients enter the simulation in accordance with the pre-defined theatre schedules and are randomly allocated a length of stay based on the length of stay distribution within the dataset. The model incorporates a virtual ITU/HDU of a size determined by the user. Upon entry patients are either admitted into a bed (if one is available) or cancelled if the unit is full. Cancelled patients exit the system immediately and are assumed not to re-enter. Admitted patients are assumed to have their surgery successfully completed and remain in the unit for the duration of their previously allocated length of stay. Figure 1 presents a screenshot of the MCS with its Excel frontend. 
The model simulates the potential activity levels during the 2012-13 financial year. It incorporates all planned downtime in the theatre schedules, due to bank holidays and the aforementioned audit sessions, but otherwise the model assumes all sessions will run as planned. The simulation commences from $1^{\text {st }}$ January 2012 to allow a warm period ahead of the commencement of the recording period, $1^{\text {st }}$ April 2012. The random nature of Monte Carlo simulation means the model can produce varying results each time it is run. In order to understand the spread of possible outcomes and capture the probability of achieving a pre-defined activity target, the simulation of the 2012-13 financial year is run 100 times over and the mean, median, maximum and minimum activity levels recorded. The percentage of iterations meeting the defined activity target is also recorded.

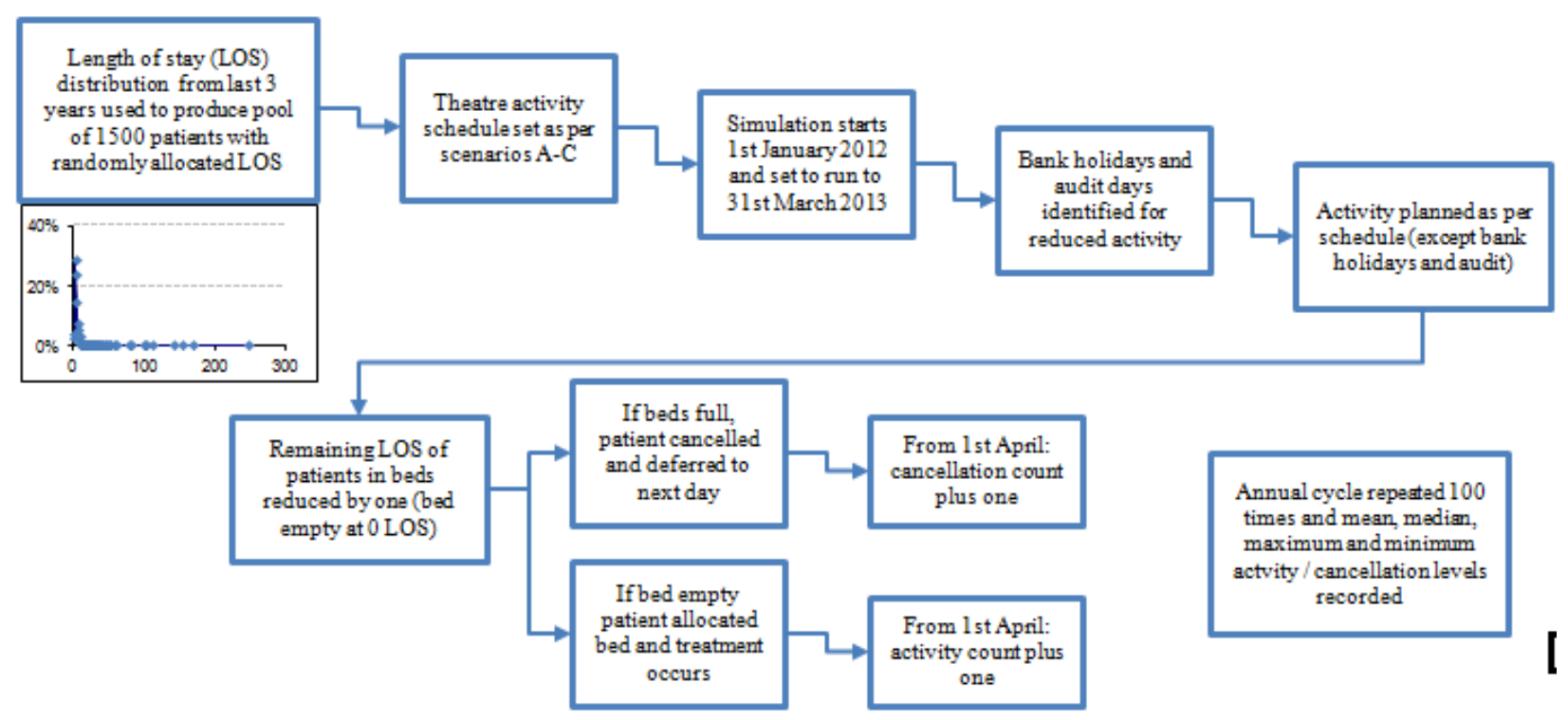

Figure 1: Modeling CITU bed capacity using MCS

\subsection{Experiments and Results}

The model has been used to simulate two different theatre schedules. Schedule A involves planning four theatre cases on a Monday, Wednesday, Thursday and Friday; and two cases on a Tuesday. Schedule B was based upon scheduling four cases Monday-Friday. These schedules were selected as they reflect the theatre schedule in use up until mid-2011 (schedule A) and the theatre schedule since that date (schedule B). The number of beds in the unit is varied between 14-21. The scenario of 14 beds for the combined ITU/HDU represents the current situation as it is assumed 2 beds are reserved for Thoracic Surgery. The target activity level per annum is 820 cases, which is the Health Board's contract for cardiac surgery. The key results from the simulations are presented in Table 1.

The results clearly demonstrate wide variation in the potential outcomes, ranging from a worst-case scenario of 615 cases per annum to a best-case scenario of 956 . There are three factors influencing the activity levels in any given year: the number of cases scheduled; the bed capacity of the unit; and chance. This clearly presents the service with a number of choices. Firstly it needs to consider the level of confidence it requires regarding the likelihood of meeting its target of 820 cases per year. This decision would need to take account of the consequences of failing the delivery target and the costs associated with achieving increased certainty. Secondly, as a given activity level may be achieved through altering the scheduling scenario and/or the bed capacity, the service needs to determine the most cost-effective arrangement which can be delivered within the existing physical limitations. Figure 2 presents the key results from the simulation influencing this decision. For each of the two scenarios it maps the likelihood of achieving the NHS base contract of 820 cardiac surgeries (based on 100 Monte Carlo iterations) by 
varying the number of beds between 14-21. For example, results show that with 20 ITU/HDU beds the likelihood of achieving the target of 820 is $95 \%$ and $100 \%$ for scenarios A and B respectively. The next stage is to cost the potential scenarios to allow the service to make these decisions in an informed way.

Table 1: Key results from Monte Carlo simulations

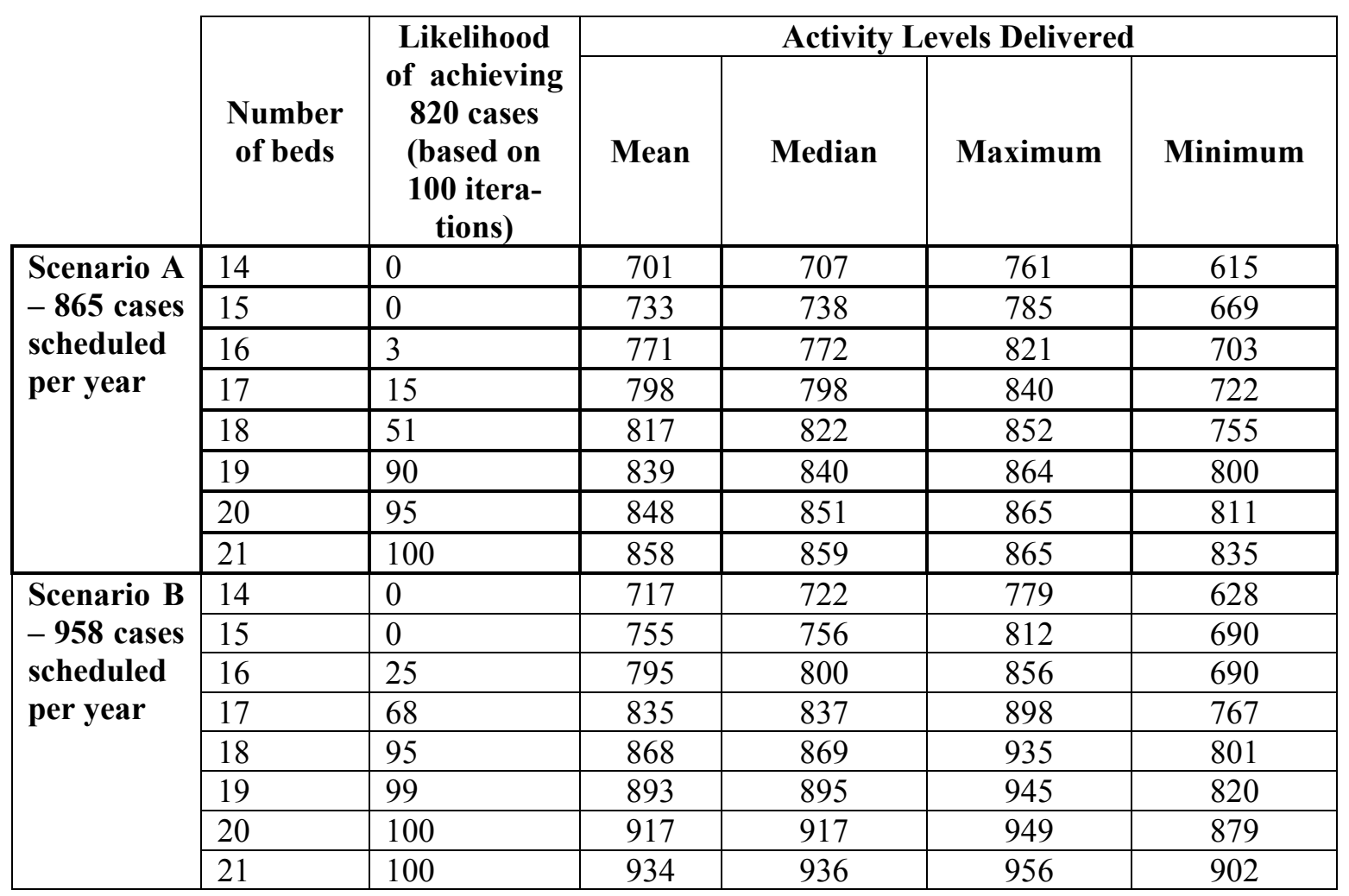

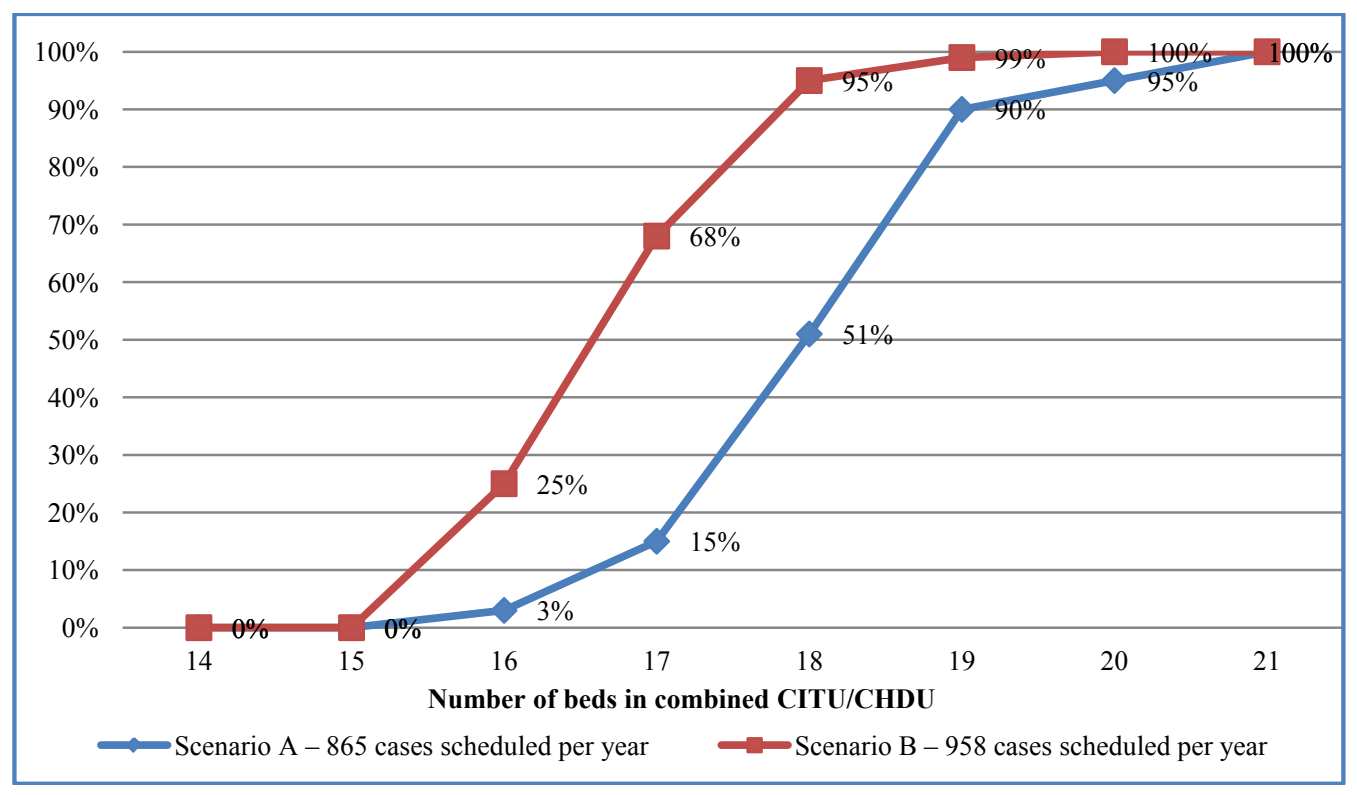

Figure 2: Likelihood of cardiac surgery service achieving 820 cases per annum (based on 100 iterations) 


\section{MODELLING PATIENT FLOW USING DISCRETE-EVENT SIMULATION}

Assuming that the transition of patients' stay through the three stages of care (i.e., from ward to ward) can be modeled independently, the modeling endeavor is likely to produce rather misleading information. With regard to the MCS model described in the earlier section, the combined ITU/HDU representation also has its drawbacks since it ignores the fact that the patients undergoing cardiac surgery are required to be admitted to the ITU for post-operative care. Thus, the unavailability of beds in ITU may trigger cancellations of cardiac surgeries, although beds may still be available in HDU.

The patient mix and coupling of their needs of different levels of care is thus likely to be important to the overall system; we have therefore created one single DES model that encompasses the resources available for the care of patients in CITU. These include three different wards - CEW, HDU and ITU each with varying number of beds (implemented as arrays of three different sets of servers), the consultant cardiac surgeons, a team of 13 highly trained nurses, two dedicated cardiac surgery theatres for cardiac surgery and one theatre for thoracic surgery. The DES model (Figure 3) is being used in the ongoing study for the realization of both the stakeholder and the research objective. The model has been created using the commercial, off-the-shelf simulation package Simul8 ${ }^{\mathrm{TM}}$ and is described below.

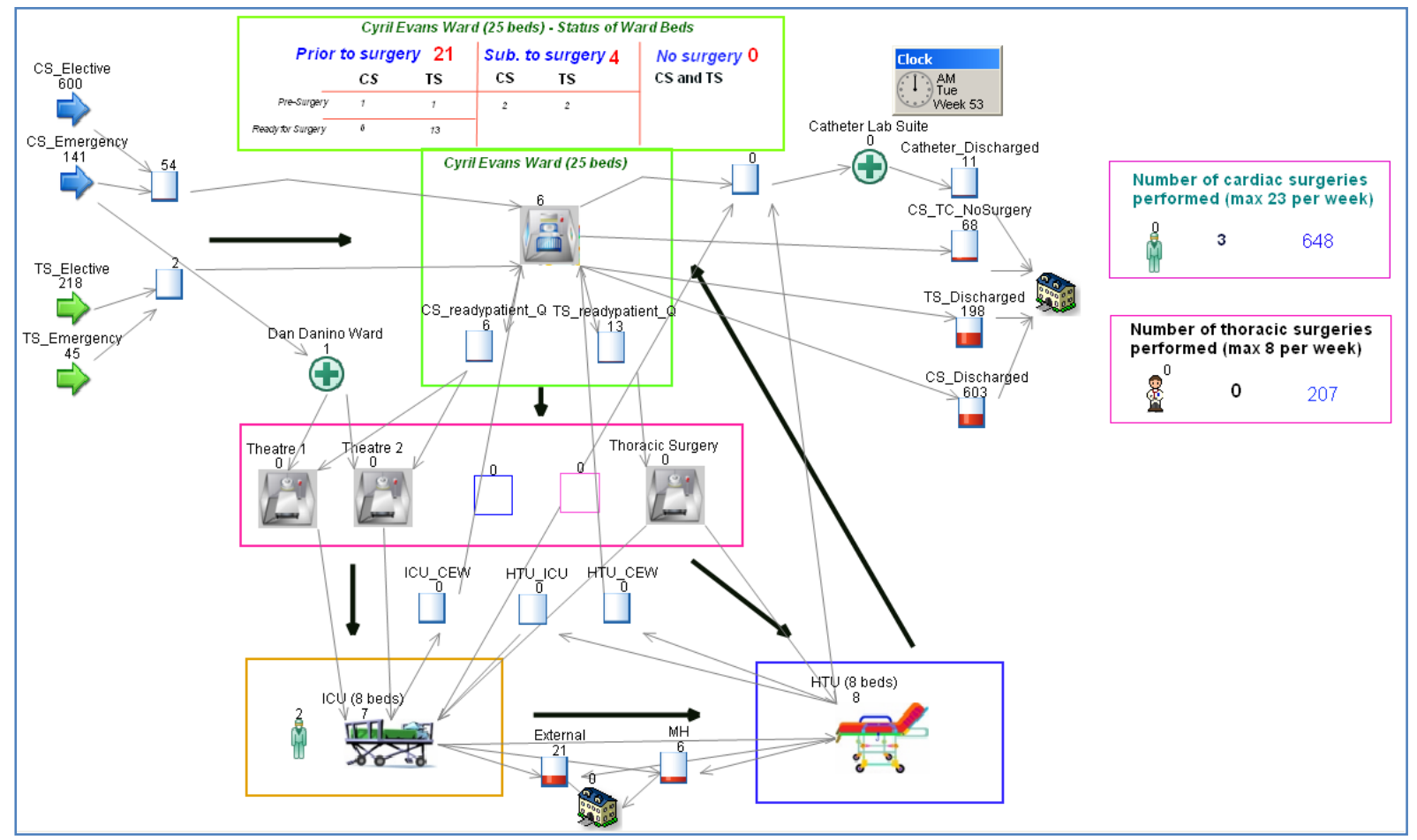

Figure 3: Modeling patient flow in CITU using DES

The model represents processes associated with both Cardiac Surgery (CS) and CardioThoracic Surgery (TS). Patients (modeled as work items) enter the CITU pathway through four entry points for TS elective patients, TS emergency patients, CS elective patients and CS emergency patients, respectively, with the majority being are admitted either to the CEW or the Dan Danino Ward (DDW). The model uses ward stay from March 2009-October 2010 to compute inter-arrival time of the patient (this data is present in an Excel worksheet that is linked with the DES model). For example, an examination of the aforementioned dataset shows that a total of 216 TS patients (195 elective TS patients; 21 emergency TS patients) and 720 CS patients (553 elective CS patients; 167 emergency CS patients) were admitted to the hospital. The dataset also informs, to a degree, the movement of the patient between wards based on the percentage of inter-ward transfers. For example, the ward stay data further drills down to the specific wards to which 
the patients were first admitted, for example, of the 167 emergency CS patients, $34 \%$ were admitted to CEW, $6.5 \%$ to the cardiac ITU (this may indicate the patients that underwent emergency cardiac surgery), $10.7 \%$ to the cardiac HTU, $19.7 \%$ to DDW, etc.

Every patient entering the system is assigned values pertaining to their LOS in the different wards. Again, this data is extracted from the ward stay data. In total there are four different LOS elements (stay in CEW prior to CS/TS surgery, stay in ITU, stay in HDU, stay in CEW subsequent to CS/TS surgery) for the following four categories of patients: CS elective patients, CS emergency patients, TS elective patients and TS emergency patients. Thus, in total there are a total of 16 different sets of LOS values that are picked up the DES model. The model differentiates between patients' stay in CEW prior to surgery and subsequent to surgery, with the former category being directed to the appropriate CS/TS patient queue "ready for surgery", and the latter being directed to the exit point (to indicate that the patient has been discharged).

The DES models three operating theatres. These theatres have resource-level constraints like assigned capacity (e.g., a limit of 20 CS with a maximum of four CS performed each day; no operations during bank holidays) and hours of operations (e.g., theatres stating at 0830 in the morning). Assuming that these constraints are adhered to, the trigger for conducting a CS is the availability of an empty bed in the ITU. In case of TS, the trigger is either the availability of an ITU or a HDU bed. Upon receiving this trigger, the operating theatre concerned "pulls" a patients from the "ready for surgery" queue. A triangular distribution defines the time the patient will stay in the operating theatre, and subsequent to the expiration of this time the patient is transferred to the ITU (in case of CS patients) or the HDU/ITU (in case of TS patients). The ward-specific LOS value determine the length of time the patient stays in the current ward, prior to making an attempt to move to the subsequent ward. The success of this move necessitates the availability of empty beds in the latter wards. In other words, since the DES models three arrays of servers (one each for general ward, ITU and HTU; the number of elements in the array is determined by their respective bed count), patients will move from one array to the other, if (a) a patient is ready to be moved to the next array (and this happens when the patient has completed the LOS required in the present array), and (b) the receiving array (e.g., HDU) has an empty element. Thus, the implementation of the model allows us to investigate the problem of bed blocking. Finally, the DES model takes into account the variable number of ITU and HDU beds during the week (refer to Section 4.2.1) and the resources that CITU shares with patients for thoracic surgery (refer to Section 4.3.2).

Some preliminary results from the DES model are now presented; we are using these results for the process of model verification and calibration. The present CITU configuration of $25 \mathrm{CEW}$ beds, eight ITU beds and eight HDU beds is simulated in a trial with 10 runs (the model is executed for one simulated year, excluding the model warm-up time of three months). At $95 \%$ confidence level, the number of cardiac surgeries performed lies in the range 619 to 667, with an average of 644. Comparing with historic data (data from 1st April 2009 to 31st March 2010 show that a total of 707 cardiac operations were performed during this period), the average result output by the model is approx. $9 \%$ lower. One reason for this is, ward data that is available to us and which is used by DES to model patients' ITU and HDU LOS, already includes the bed blocking component. However, the logic in the DES model further adds to the LOS since, for example, HTU beds may be unavailable and a patient entity may have to remain in ITU. This further magnifies the bed blocking component, and contributes to a lower number of cardiac surgeries being reported by the DES model. We aim to further calibrate the model so that the difference is reduced to $+/-5 \%$.

As mentioned earlier, the purpose of this model is twofold: (a) to investigate the bed configuration that would allow the Cardiac Center to achieve the target of $820 \mathrm{CS}$ each year, and (b) experiment with alternate bed management strategies with the objective of reducing bed blocking. This is an ongoing work and we expect to present the results of the DES study in a future publication. 


\section{CONCLUSION}

The paper has investigated the problem of bed blocking through a case study being conducted at the Cardiac Intensive Care Unit (CITU) at Morriston Hospital, Wales (UK). The number of cardiac surgeries performed in the Cardiac Centre is constrained by the availability of resources (i.e. physically vacant beds supported by skilled clinical teams), and the bottlenecks that are present in the existing system. With regard to the latter, bed blocking has been identified as a significant bottleneck. In this paper we have identified two objectives, both of which aim to reduce the impact of bed blockers in specialized and integrated care units, but are based on different underlying premises - shortage of beds (and thus the requirement to find the optimal number of beds) versus alternate bed management strategies (considering that the number of beds cannot be increased in the short run. The paper has focused on the first objective and has presented a MCS study that has experimented with different bed numbers. Although this approach is useful in accounting for bed blockers, an extension of the study could be to experiment with different rates of bed blocking. However, to enable these experiments to be conducted, the actual stay of patient has to be divided into two components, their clinical length of stay and stay due to bed blocking, and data collected for each. This is required since actual stay $=$ clinical length of stay + stay due to bed blocking. This is planned for the future.

The paper has also outlined the need for a detailed DES model for experimenting with bed management strategies (second objective) and has presented an overview of the model. Future work will focus on experimenting with the DES model to further refine the estimate of the optimal bed numbers provided by the MCS study and to investigate different bed management strategies like keeping beds empty to reduce congestion in the system. As in most blocking situations, one would expect that there is little to be gained by filling the less constrained parts of the system to capacity and forming a traffic jam and that the largest throughput might well be achieved by allowing congestion in later parts of the process to force restraint in initial admission. Thus, empty beds need not be an indicator of inefficiency, but of the converse.

\section{REFERENCES}

ABMUHB. 2012. Abertawe Bro Morgannwg University Health Board. http://www.abm.wales.nhs.uk/ [Accessed April 11, 2012].

ABM UHB INFO. 2010. Data Pertaining to CCU Wards (CEW, ITU, HDU) for Cardiac Surgery and Thoracic Surgery - period 1st April 2009 to 31st October 2010. ABM University Health Board - Information Directorate.

Bagust, A., M. Place, and J. W. Posnett. 1999. "Dynamics of Bed Use in Accommodating Emergency Admissions: Stochastic Simulation Model." BMJ 319:155-158.

Brailsford, S. C., P. R Harper, B. Patel, and M. Pitt. 2009. "An Analysis of the Academic Literature on Simulation and Modelling in Health Care." Journal of Simulation 3(3):130-140.

Brooks, R., S. Robinson, and C. Lewis. 2001. Simulation and Inventory Control - Operational Research Series. Hampshire, UK: Palgrave.

Chahal, K. and T. Eldabi. 2008. "Applicability of Hybrid Simulation to Different Modes of Governance in UK Healthcare." In Proceedings of the 2008 Winter Simulation Conference, Edited by S. J. Mason, R. R. Hill, L. Mönch, O. Rose, T. Jefferson, and J. W. Fowler, 1469 - 1477. Piscataway, New Jersey: Institute of Electrical and Electronics Engineers, Inc.

Coid, J. and P. Crome. 1986. "Bed Blocking in Bromley." BMJ 292:1253-1256.

El-Darzi, E., C. Vasilakis, T. Chaussalet, and P. H. Millard. 1998."A Simulation Modelling Approach to Evaluating Length of Stay, Occupancy, Emptiness and Bed Blocking in a Hospital Geriatric Department." Health Care Management Science 1(2):143-149.

Fone, D., S. Hollinghurst, M. Temple, A. Round, N. Lester, A. Weightman, K. Roberts, E. Coyle, G. Bevan, and S. Palmer. 2003. "Systematic Review of the Use and Value of Computer Simulation Modelling in Population Health and Health Care Delivery." Journal of Public Health Medicine 25(4): 325-335. 
Heslop, L., B. Gardner, D. Athan, D. Diers., and C. Taylor. 2004. "Researching Hospital Patient Data to Enhance Operational Management." Health Information Management 32(1):13-16.

ICS. 2011. What is Intensive Care? Intensive Care Society (ICS) website. http://www.ics.ac.uk/patients_relatives/what_is_intensive_care_[Accessed April 11, 2012].

Jun, J. B., S. H. Jacobson, and J. R. Swisher. 1999. "Application of Discrete-event Simulation in Health Care Clinics: A Survey." Journal of the Operational Research Society 50(2):109-123.

Katsaliaki, K. and N. Mustafee. 2011. "Applications of Simulation Research within the Healthcare Context." Journal of the Operational Research Society 62(8):1431-1451.

Leemis, L. and S. Park. 1994. Discrete Event Simulation: A First Course. http://coins.csrlab.org/imamu/akoubaa/cs433/Textbooks/ [Accessed April 17, 2012]

MCC. 2006. Pre-Operative Information Booklet for Cardiac Patients. Moriston Hospital Cardiac Centre (MCC) website. http://www.swansea-tr.wales.nhs.uk/cardiac/ [Accessed October 26, 2011].

Mustafee, N., F. Hughes, K. Katsaliaki, and M. D. Williams. 2011. "Simulation-based Study of Hematology Outpatient Clinics with Focus on Model Reusability." In Proceedings of the 2011 Winter Simulation Conference, Edited by S. Jain, R. R. Creasey, J. Himmelspach, K.P. White, and M. Fu, 1178 1189. Piscataway, New Jersey: Institute of Electrical and Electronics Engineers, Inc.

Namdaran, F., C. Burnet, and S. Munroe. 1992. "Bed Blocking in Edinburgh Hospitals." Health Bulletin 50(3):223-227.

Pidd, M. 2004. Computer Simulation in Management Science. 5th edition. Chichester, UK: John Wiley.

RCS. 2010. Death Rates for NHS cardiac surgery 25\% better than European Average, says First International Audit. The Royal College of Surgeons of England (RCS) webpage. http://www.rcseng.ac.uk/news/death-rates-for-nhs-cardiac-surgery-25-better-than-european-averagesays-first-international-audit [Accessed April 11, 2012).

Rubinstein, R.Y. 1981. Simulation and the Monte Carlo Method. New York, USA: John Wiley \& Sons.

Utley, M., S. Gallivan, K. Davis, P. Daniel, P. Reeves, and J. Worrall. 2003. "Estimating Bed Requirements for an Intermediate Care Facility." European Journal of Operational Research 150(1):92-100.

\section{AUTHOR BIOGRAPHIES}

NAVONIL MUSTAFEE is a lecturer in Operations Management and Information Systems in the School of Business and Economics, Swansea University (UK). His research interests are in simulation methodologies, healthcare simulation, parallel and distributed simulation, technology adoption and diffusion, and grid computing. His e-mail address is n.mustafee@swansea.ac.uk and his web page is http://sites.google.com/site/navonilmustafee/.

LEE DAVIES is a Performance Improvement Manager at the Delivery and Support Unit, NHS Wales. His email address is lee.davies3@wales.nhs.uk.

TERRY LYONS is the Wallis Professor of Mathematics and Director of Oxford-Man Institute of Quantitative Finance, University of Oxford. His email address is tlyons@maths.ox.ac.uk.

MARK RAMSEY is a Consultant Cardiologist and Clinical Director, Regional Services - Abertawe Bro Morgannwg University Health Board. His email address is mark.ramsey@wales.nhs.uk.

PAUL REES is a Senior Clinical Nurse, Cardiac Care Unit, Regional Services - Abertawe Bro Morgannwg University Health Board. His email address is paul.rees3@wales.nhs.uk.

MICHAEL D. WILLIAMS is a Professor in the School of Business and Economics at Swansea University in the UK. He holds a BSc from the CNAA, an MEd from the University of Cambridge, and a PhD from the University of Sheffield. He can be contacted at: m.d.williams@swansea.ac.uk. 